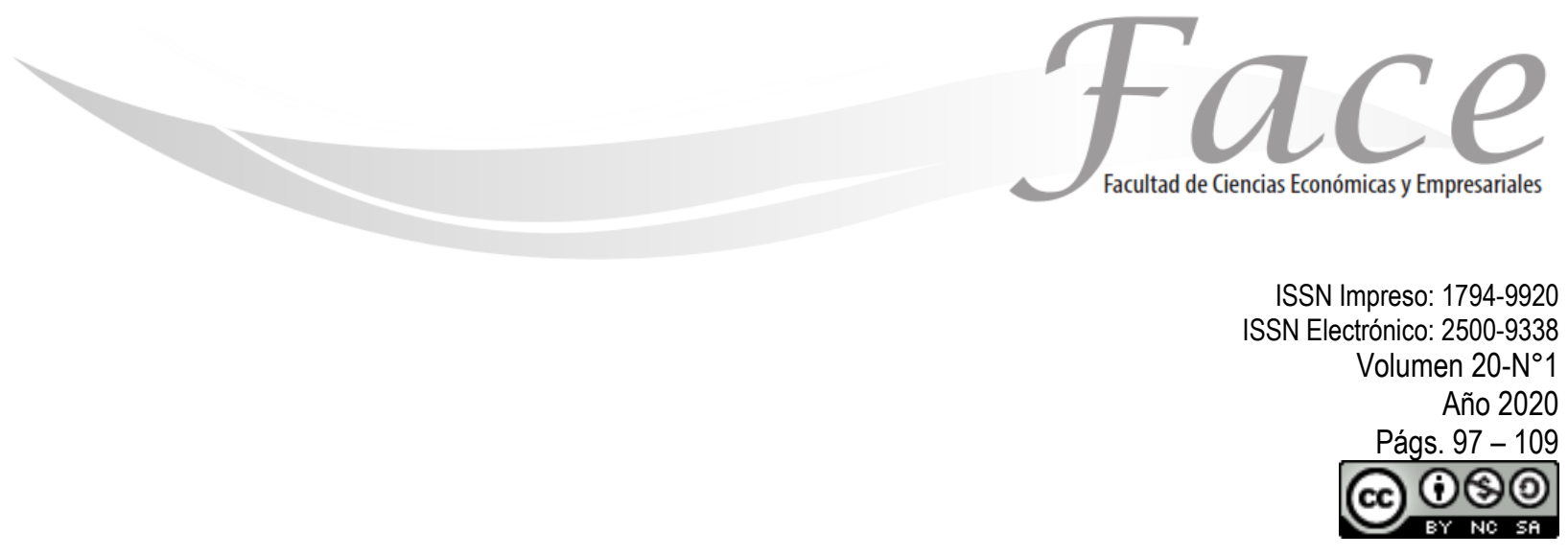

\title{
LOS EFECTOS DE LA INFLACIÓN SOBRE LA TASA DE INTERÉS EN COLOMBIA 2005 - 2019
}

\author{
Antonio María Martínez Reina * \\ https://orcid.org/0000-0002-9312-842X
}

Fecha de Recepción: Abril 15 de 2020

Fecha de Aprobación: Julio 22 de 2020

\section{Resumen:}

El objetivo de este trabajo fue analizar la evidencia empírica de los efectos de la inflación en la tasa de interés a largo plazo en Colombia en el periodo 2005 - 2019. Es el resultado de una investigación espontanea del autor con el fin de hacer un trabajo empírico con datos oficiales para probar la consistencia de la teoría económica de la hipótesis de Fisher. Con información del Banco de la Republica de Colombia para el caso de la tasa de interés y el departamento administrativo nacional de estadística DANE para la inflación. Se usaron estadísticas descriptivas, medidas de dispersión, además con técnicas econométricas como las pruebas de raíces unitarias y la cointegración, se determinó el grado de integración de las dos variables, también se hicieron análisis de casualidad por el método de Granger. Como marco teórico se usó la hipótesis de Fisher que plantea una relación entre los cambios futuros en la tasa de interés nominal como consecuencia de la inflación presente y futura. Los resultados permiten apreciar la relación alta en estas dos variables, como también la integración, lo que permite concluir que la inflación determina el comportamiento futuro de la tasa de interés en Colombia.

Palabras clave: Integración, precios, tendencia, tasa de interés, inflación, oferta monetaria.

\footnotetext{
* Doctor en Ciencias Económicas, Universidad Autónoma De Baja California Sur-México; Magister en Ciencias Agrarias de la Universidad NacionalColombia; Economista de Universidad Pedagógica y Tecnológica de Colombia-Colombia. Afiliación Institucional: Corporación Colombiana de Investigación Agropecuaria-AGROSAVIA. C.I. Turipaná. Cereté, Córdoba-Colombia. Contacto: amartinezr@agrosavia.co
} 


\title{
THE EFFECTS OF INFLATION ON THE INTEREST RATE IN COLOMBIA 2005 - 2019
}

\begin{abstract}
:
The objective of this work was to analyze the empirical evidence of the effects of inflation on the long-term interest rate in Colombia in the period 2005 - 2019. It is the result of a spontaneous investigation by the author to do an empirical work with official data to test the consistency of the economic theory of Fisher's hypothesis. With information from the Banco de la Republica de Colombia for the case of the interest rate and the national administrative department of statistics DANE for inflation. Descriptive statistics, dispersion measures were used, in addition to econometric techniques such as unit root tests and cointegration, the degree of integration of the two variables was determined, chance analysis was also done by the Granger method. As a theoretical framework, the Fisher hypothesis was used, which proposes a relationship between future changes in the nominal interest rate because of present and future inflation. The results allow us to appreciate the high relationship in these two variables, as well as integration, which allows us to conclude that inflation determines the future behavior of the interest rate in Colombia.
\end{abstract}

Keywords: compliance, formal duties, tax, income, value added.

\section{OS EFEITOS DA INFLAÇÃO NA TAXA DE JURO NA COLÔMBIA 2005 - 2019}

\section{Resumo:}

O objetivo deste trabalho foi analisar a evidência empírica dos efeitos da inflação sobre a taxa de juros de longo prazo na Colômbia no período 2005-2019. É o resultado de uma investigação espontânea do autor para fazer um trabalho empírico com oficiais dados para testar a consistência da teoria econômica da hipótese de Fisher. Com informações do Banco de la Republica de Colombia para o caso da taxa de juros e do departamento administrativo nacional de estatísticas DANE para a inflação. Foram utilizadas estatísticas descritivas, medidas de dispersão, além de técnicas econométricas como testes de raiz unitária e cointegração, foi determinado o grau de integração das duas variáveis, análise de chance também foi feita pelo método de Granger. Como referencial teórico, foi utilizada a hipótese de Fisher, que propõe uma relação entre variações futuras da taxa de juros nominal em função da inflação presente e futura. Os resultados nos permitem avaliar a alta relação entre essas duas variáveis, bem como a integração, o que nos permite concluir que a inflação determina o comportamento futuro da taxa de juros na Colômbia.

Palavras-chave: compliance, obrigações formais, imposto, renda, valor adicionado 


\section{INTRODUCCIÓN:}

Uno de los grandes debates en materia de política económica es el manejo de los precios y sus efectos negativos sobre la economía en su conjunto y en particular sobre el nivel de vida de las personas. Las respuestas y la participación en este debate vienen principalmente de dos vertientes una que afirma que la inflación se produce cuando el dinero circulando es mayor que el valor de la producción, planteamiento de los monetaristas (Friedman et al 1963). Por su parte hay quienes que piensan que los excesos en la cantidad de dinero no producen aumentos constantes y ascendentes de los precios porque cualquier aumento de la oferta monetaria bajaría la tasa de interés y aumentaría la demanda de dinero, en consecuencia, la inflación no es un fenómeno monetario (Keynes 1984).

Las relaciones entre la tasa de interés nominal y la inflación para el caso colombiano no han sido estudiadas por lo menos para los últimos 10 años. Es de anotar que quien propuso por primera vez esta relación fue Fisher 1930, según este autor encontró que se da una transmisión del crecimiento del nivel general de precios en forma total o parcial sobre la tasa de interés nominal y se conoce como "el Efecto Fisher" (1930). Este concepto ha revestido importancia tanto en la teoría económica como en el manejo de la inflación por parte del banco central para el caso colombiano. En caso de aceptarse la premisa de Fisher, se podría considerar que los cambios en la cantidad de dinero en circulación en el largo plazo no afectarían a las variables reales como el empleo, valor de la producción nacional y el consumo que se conoce en la teoría económica como neutralidad del dinero (Hayek 1933).
Para el caso colombiano sobre la evidencia empírica de la neutralidad del dinero y manejo de la inflación se encuentran algunas referencias como las de Uribe 1994 a través de la técnica de la estimación por mínimos cuadrados se relaciona el crecimiento económico con la inflación para

medir sus efectos, se usaron series de tiempo para el periodo 1952-1992. Los resultados dan cuenta de los efectos negativos de la inflación sobre el crecimiento expresado en bajas en la productividad de los factores de producción y altos costos de producción.

Por su parte Dornbusch (2009) reafirma esta situación cuando dice que, a largo plazo, cuando sean producido todos los ajustes, un aumento de la inflación se refleja totalmente en los tipos de interés nominales. Considero esta la premisa que se trata de desarrollar en el presente trabajo con datos de instituciones oficiales y de dominio público. El estudio y análisis de esta relación de variables como son la transmisión de las tasas de interés nominal conocida como efecto Fisher.

La teoría propone que, en el largo plazo, la inflación trae efectos en la tasa de interés es decir que primero ocurren los cambios en el nivel general de precios y luego se transmiten a la tasa de interés nominal. Los tipos de interés suben en la misma cuantía que la inflación Dornbusch (2009). Esta relación es la que se pretende probar con este ejercicio econométrico con datos de instituciones oficiales como el Banco de la Republica de Colombia y el Departamento Nacional de Estadísticas DANE.

Galindo (1994), llevó a cabo un estudio llamado "Política económica e inflación: periodo 1988-1994. demostró que los desequilibrios cambiarios, pueden traer graves consecuencias en el manejo de la inflación, es así, que un desequilibrio de un punto puede incrementar la tasa de inflación en casi dos puntos porcentuales al cabo de un año en una economía con cierto grado de indexación como la colombiana. La inflación se convierte entonces en un mecanismo natural de corrección de desviaciones de la tasa de cambio real con respecto a su nivel de largo plazo. 
La evidencia empírica del efecto Fisher para el caso colombiano fue analizado por Cárdenas et al 2001 con una serie de datos para el periodo 1980-2000 de las variables tasa de interés nominal y de la tasa de inflación medidos por el IPC. Realizaron un trabajo con la formulación de un modelo econométrico con pruebas de integración de variables a pesar de que las series presentan raíz unitaria, están integradas en el largo plazo. Los resultados permiten inferir que los incrementos en los precios se transmiten a la tasa de interés nominal. En este caso se cumple la teoría de la neutralidad del dinero.

Otro trabajo en esta misma dirección fue el realizado por Ávila 2013 quien usó series de tiempo en un periodo 2000- 2010 para estudiar los efectos de la inflación en la demanda de dinero, las variables que relacionó fueron la oferta Monetaria $\mathrm{M}_{1}$, el PIB, la tasa de interés nominal. La técnica que usó fue la de los mínimos cuadrados ordinarios y el marco teórico Monetarista. Concluye que las variables como PIB y el nivel de precios son las que más influencia tienen sobre la demanda de dinero.

El objetivo de este trabajo es validar la teoría de la neutralidad de dinero en el caso colombiano con la información disponible con el uso de la técnica econométrica de la cointegración con datos de la economía colombiana.

La pregunta de investigación fue: ¿La tasa de interés nominal en el largo plazo se ve influenciada por el crecimiento de los precios?

\section{METODOLOGÍA:}

Para demostrar la evidencia del efecto Fisher se usaron las estadísticas disponibles sobre inflación que publica el DANE y en el caso de la tasa de interés las disponibles en el Banco de la Republica de Colombia. Las series trimestrales comprendieron el periodo enero de 2005 a diciembre de 2019. Las variables que se analizaron fueron la inflación medida a través del índice de precios al consumidor del DANE y el certificado de deposito a termino DTF a 90 días para el caso de la tasa de interés nominal. Con el fin de conservar la pureza de los datos no se realizó ningún tipo de transformación y se tomaron como datos originales.

Se planteó como modelo general para medir el efecto de la inflación sobre la tasa de interés (efecto Fisher) en la economía colombiana como se presenta a continuación:

$R_{t}=\alpha_{0}+\beta_{1} x_{t-1}+\beta_{2} x_{t-2}+\beta_{3} x_{t-2}+\beta_{3} x_{t-1}+\mu(1)$

Donde:

$Y_{t}=$ Movimiento de la serie a través del tiempo

$B=$ Parámetros que explican los movimientos del tiempo en un periodo $t$.

$\mathrm{xt}_{-1}=$ Precio del tiempo presente con relación al precio del periodo anterior.

$\mu=$ Termino de perturbación.

Para el caso de lo que se quiere probar que es si la tasa de interés se ve influenciada por la inflación en el largo plazo se presenta mediante la siguiente ecuación:

$$
R=\alpha+\beta x+\mu
$$

Donde:

$\mathrm{R}=\mathrm{La}$ tasa de interés nominal

$\mathrm{x}=$ Tasa de inflación

$\mu=$ Termino de perturbación.

Una vez se bajaron las series de las páginas web como del Banco de la Republica de Colombia, se procedió a equiparar para que quedaran en periodos trimestrales en consideración a que las tasas DTF estaban mensualmente. 
Igualmente se usó la prueba de corrección de error

Una vez se tuvieron las series expresadas por trimestres del periodo 2005 - 2019 se calcularon estadísticas descriptivas y algunas medidas de tendencia central para analizar las característicasde las series, además se realizaron análisis de correlación.

La primera inquietud que surgía era saber si se estaba trabajando con series estacionarias por lo que se hizo el análisis gráfico, los correlogramas, las pruebas de raíces unitarias y se aplicado la prueba de Engle y Granger (1987). Se estableció que las series en niveles no son estacionarias solo la tasa de interés DTF fue significativa al $97 \%$. Se hizo la prueba en primeras diferencias y las series resultaron estacionarias lo que quiere decir que están integradas de grado dos. Lo que significa que las dos series deambulan en el tiempo en la misma dirección sin que se encuentren o crucen en ningún momento del tiempo. El paso siguiente fue hacer el análisis de cointegración para mostrar el grado de integración de las variables en el tiempo. En otras palabras, si comparten una secuencia estocástica común es decir que mantienen la misma relación a lo largo del tiempo. Existe un vector $\beta=\left(\beta_{1}-\beta_{n}\right)$ en el cual la combinación lineal $\beta_{1} X_{1}+\beta_{2} X_{2}+\beta_{3} X_{3}$ que es integrada del orden $I(d-b)$ donde $b>0$. (Johansen 1988).

Cuando se construyeron los archivos planos en Excel, con las series de tasa de interés y la inflación (IPC) se procedió a hacer las pruebas antes mencionadas mediante el uso del programa econométrico EViews versión ocho en la función Método de descomposición de la serie, que permite determinar el ciclo y la estacionalidad de la serie, la cual se realizó mediante la prueba de Dickiey y Fuller (1981) y prueba de causalidad de Granger con el fin de determinar si el comportamiento de la tasa de interés nominal estaba determinado por la inflación. para probar la transmisión de los cambios en la inflación en la tasa de interés en el largo plazo considerando el vector $R_{t}$ de la tasa de interés observada en un mismo periodo de tiempo t. Lo mismo para la inflación. En este momento se establece que solo los movimientos de la tasa de interés se deben al comportamiento de la inflación, solo dependen del tiempo t y están integradas del orden 1 (1), es decir que las series de las diferencias en tanto en la tasa de interés como en la inflación en los dos periodos consecutivos del tiempo, $\quad \Delta R_{1}=r_{t} r_{t-1}$ son estacionarias, los precios observados en un periodo $t$ dependen de los observados en los periodos anteriores $Y=\beta_{0}+\beta_{1} \Delta_{x} t+\beta_{2} \mu t-1+\varepsilon_{t}$ (Engle $\mathrm{y}$ Granger 1987)

\section{RESULTADOS Y DISCUCIÓN:}

Con las series ya construidas tanto de la tasa de interés nominal como de la inflación medida en el IPC Se construyeron dos series de datos, una por cada variable, para el periodo enero de 2005 diciembre de 2019 en total 61 casos y se graficaron para observar el comportamiento a través del tiempo. Ver Figura 1. 
Figura $\mathrm{N}^{\circ} 1$ Series de tasa de interés nominal DTF e inflación en Colombia enero de 2005 - diciembre de 2019.

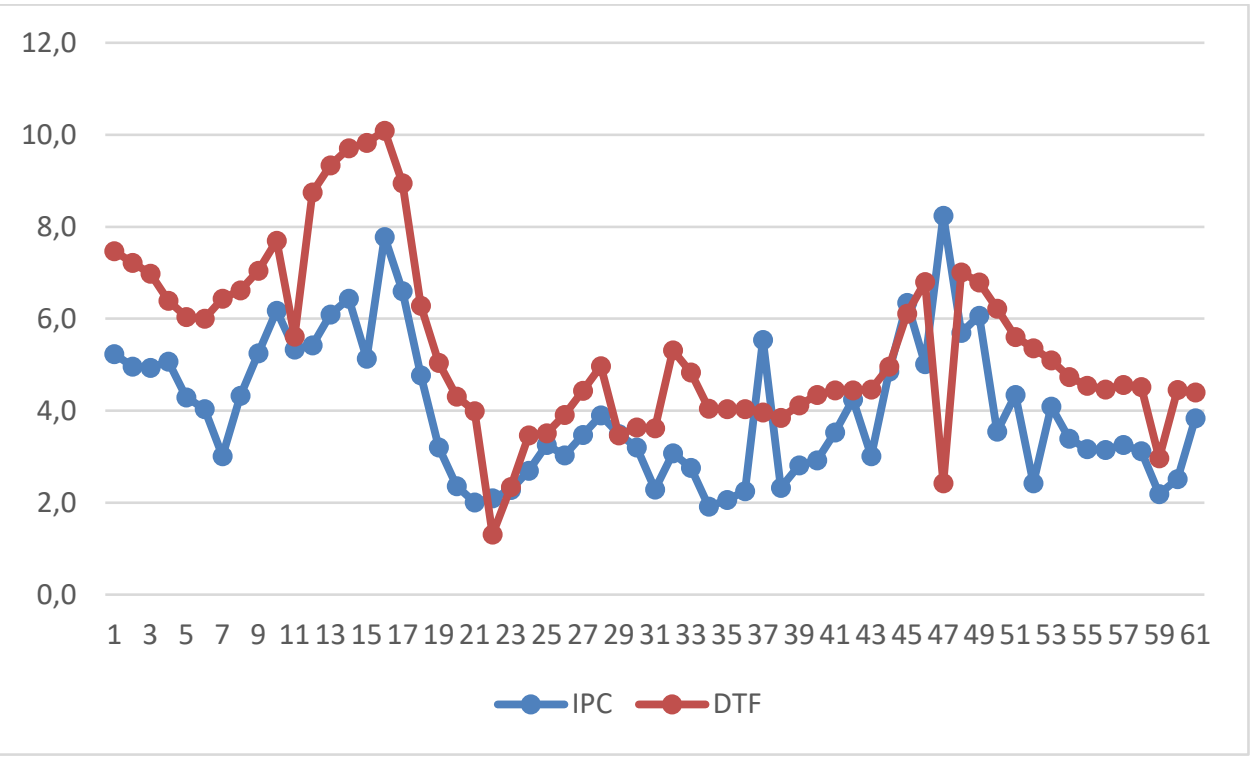

Fuente: Elaboración propia con base en SIPSA Dane 2020

La Figura 1 presenta la evolución tanto de la tasa de interés como de la inflación en Colombia. Se aprecia que todas las series presentan una tendencia creciente uniforme sin cambios en el tiempo y siempre en la misma dirección durante el periodo analizado como un indicio que las series son estacionarias en primeras diferencias.
Como un referente y para reafirmar el carácter estable que presentan los datos se hicieron cálculos de estadísticas descriptivas las cuales se presentan en la tabla1.

Tabla №1. Estadísticas descriptivas del ipc cdt de Colombia 2005 - 2019.

\begin{tabular}{lcc}
\hline \multicolumn{1}{c}{ Variable } & IPC & DTF \\
\hline Media & 4 & 5,3 \\
Mediana & 3,5 & 4,8 \\
Moda & 2,3 & 4 \\
Mínimo & 1,9 & 1,3 \\
Máximo & 8,2 & 10,1 \\
Desviación estándar & 1,4 & 1,9 \\
Coeficiente de variación & 0,3 & 0,35 \\
Varianza & 2,26 & 3,6 \\
Correlación & 0,68 & 0,68 \\
\hline
\end{tabular}

Fuente: Cálculos propios con base en Banco de la Republica

y DANE 2020 
El diagnostico de los datos se puede apreciar en la tabla 1. En primer lugar, las medidas de tendencia central permiten inferir que los datos presentan una distribución normal con unos valores promedio de 4 para el caso del índice de precios al consumidor como variable utilizada para medir la inflación y 5,3 para el DTF como medida de la tasa de interés nominal. La moda para el IPC y el DTF fue de 2,3 y 5,3 respectivamente. Se pudo apreciar que los datos presentan baja dispersión lo cual se puede establecer al observar el coeficiente de variación que en los dos casos se ubica en el $30 \%$ siendo mayor para el caso del DTF en cinco puntos. Por su parte se encontró un coeficiente de correlación alto del 0,68 y una varianza de 2,26 para el caso del IPC y 3,6 para el caso del DTF. Lo anterior da mucha confianza en cuanto a consistencia de la serie. Se deja claro que la serie no fue generada sino tomada de las estadísticas oficiales de dominio público.
Se deja claro que a pesar de que las series se encuentran altamente correlacionadas con un coeficiente del $68 \%$, no se puede asegurar que existe causalidad por lo menos en este momento del análisis por lo que es necesario realizar otras pruebas que conduzcan a afirmar o negar esta situación.

Continuando con la realización de las pruebas para determinar en primer lugar la relación de una variable frente a la otra, es decir la relación que existe entre el IPC como tasa de inflación y el DTF como tasa de interés nominal se hace el análisis gráfico el cual se presenta en la figura número 2 para el caso del IPC.

\section{Figura $\mathrm{N}^{\circ} 2$. Análisis grafico de las series de IPC para Colombia enero de 2005 - diciembre 2019.}

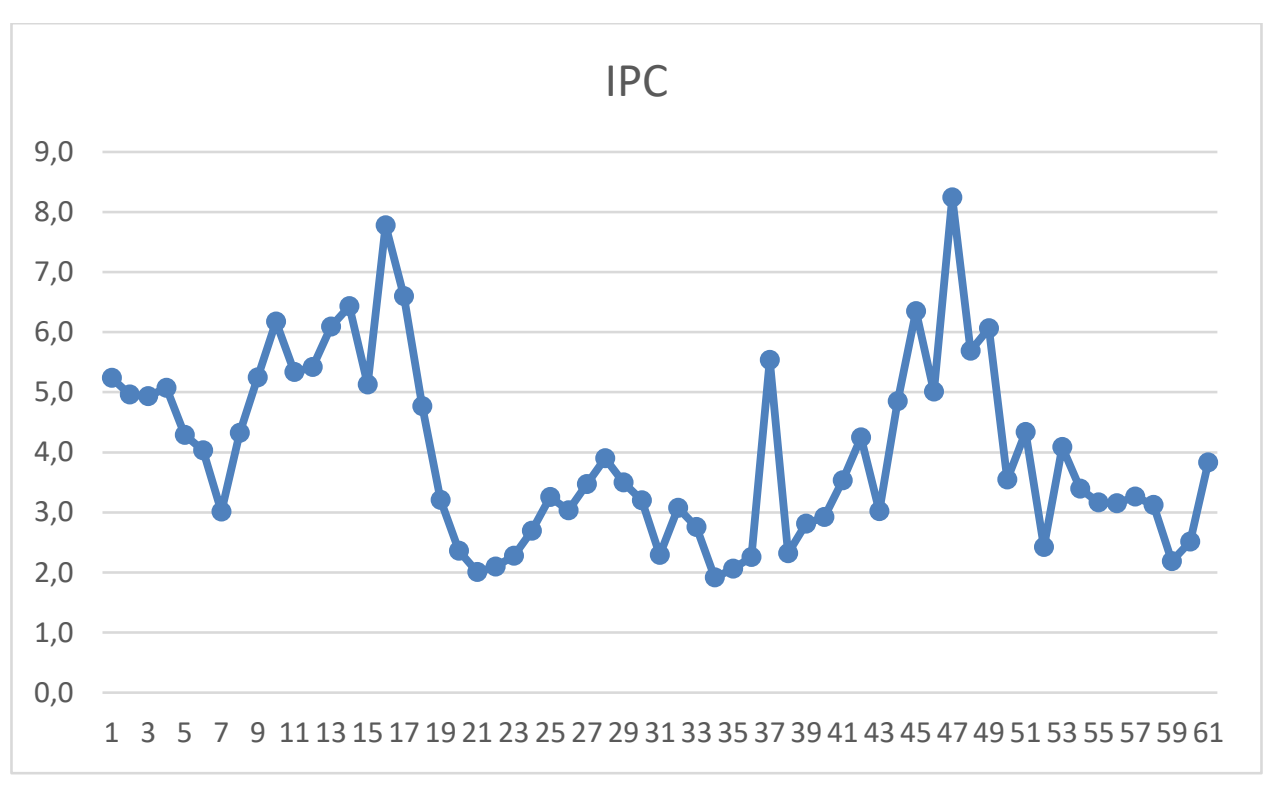

Fuente: Elaboración propia con base en Dane 2020.

La figura número 2 deja apreciar la serie de la variable IPC en datos originales se observa algunos periodos están muy por debajo de la línea de tendencia como es el caso en el tercer trimestre del año 2012 y la máxima, por su parte el pico más alto de la serie lo presentó en el primer trimestre de 2017. Se aprecia también 
intervalos muy grandes entre el valor mínimo y el máxima 1,9 y 8,2 respectivamente, sin embargo, las variaciones totales fueron del $30 \%$ que se consideran normales en este tipo de información.

Para la variable tasa de interés nominal representada en el DTF el comportamiento según el análisis grafico se puede observar en la figura $\mathrm{N}^{\circ} 3$.
Por su parte cuando se realizaron los correlogramas mostraron tanto en el caso del IPC como en el DTF que, aunque muy cercano a cero presentaban raíces unitarias en niveles y solo se pudo demostrar que las dos series son estacionarias en primeras diferencias.

\section{Figura $\mathrm{N}^{\circ} 3$. Análisis grafico de las series de DTF para Colombia} enero de 2005 - diciembre 2019.

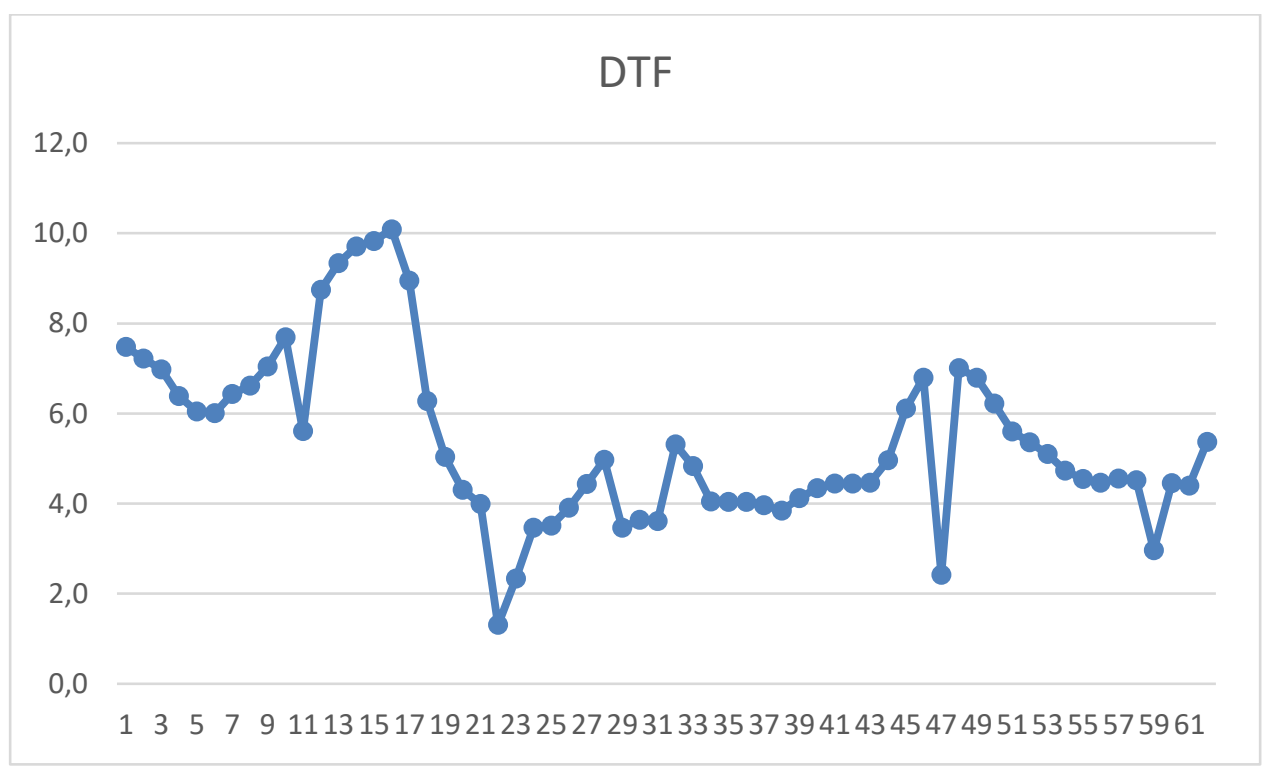

Fuente: Elaboración propia con base en Banco de la Republica.2020.

La figura $\mathrm{N}^{\circ} 3$ deja apreciar la serie de la variable DTF en datos originales se observa algunos periodos están muy por debajo de la línea de tendencia como es el caso en el segundo trimestre del año 2010 y el tercer trimestre de 2016 el valor máximo en la tasa de interés nominal lo presenta en el primer trimestre de 2009. Se aprecia también intervalos muy grandes entre el valor mínimo y el máxima 1,3 y 10,1 respectivamente, sin embargo, las variaciones totales fueron del $35 \%$ que se consideran normales en este tipo de información.
Es de anotar que el caso de los CDT resultó estacionarias a un nivel de confianza del $93 \%$ por lo que no satisfizo el del $95 \%$ y por esta razón se usaron primeras diferencias y en estas circunstancias las dos series son estacionarias.

Las pruebas de raíces unitarias se hicieron bajo el criterio de Dickey-Fuler (1981) con el fin de comprobar una vez más la estacionariedad de las series, es decir si el comportamiento se mantiene en el tiempo. La salida de las pruebas de raíces unitarias se presenta en la tabla $\mathrm{N}^{\circ} 2$. 


\section{Tabla $N^{\circ} 2$ Salidas de las pruebas de raíces unitarias de Dickey-Fuller en las series de ipc y cdt 2005 - 2019}

\begin{tabular}{lcc}
\hline Variable & $\begin{array}{c}\text { Dickey-Fuller } \\
\text { Primeras diferencias }\end{array}$ & Prob \\
\hline IPC & -1.129 .812 & 0.0000 \\
DTF & -9.860 .059 & 0.0000 \\
\hline
\end{tabular}

Fuente: Elaboración propia con base en Dane y Banco de la Republica.

La tabla $\mathrm{N}^{\circ} 2$ permite apreciar que cuando se realizó la prueba de raíces unitarias Dickey-Fuller para el caso de las variables IPC y DTF como medidas de la inflación y la tasa de interés nominal. De acuerdo con los resultados no se detectó presencia de raíces unitarias en primeras diferencias en ninguna de las dos variables lo que quiere decir que tanto la tasa de interés como la inflación son I(1)a un nivel de significancia del $5 \%$. Lo que significa que las dos series mantienen la misma tendencia en el deambular en el tiempo y son independientes entre sí lo que permite dar el siguiente paso que es el análisis de cointegración.

Pero antes de realizar las pruebas de cointegración con el fin de estar seguros de la consistencia del modelo como ajuste y prueba de mulcolinealidad se estimaron los parámetros usando como variable dependiente la tasa de interés y como variable independiente la inflación, lo cual dio como resultado los datos de la Tabla $\mathrm{N}^{\circ} 3$.

Tabla N³ Resultados de la estimación de parámetros entre la tasa de interés y la inflación.

\begin{tabular}{lllcl}
\hline Variable & Coeficiente & Std. Error & t-Statistic & Prob. \\
\hline C & 1.926 .419 & 0.514195 & 3.746 .478 & 0.0004 \\
IPC & 0.858091 & 0.120488 & 7.121 .774 & 0.0000 \\
Durwin watson & \multicolumn{2}{c}{1,4} & & \\
$\mathrm{R}^{2}$ & \multicolumn{2}{c}{0,46} & & \\
\hline
\end{tabular}

Fuente: Elaboración propia con datos de Banco de la Republica y Dane.
Se aprecia en la tabla $N^{\circ} 3$ que la estimación de la función presenta robustez en la medida que presenta una bondad de ajuste medida en el coeficiente de determinación cercano al 50\%, además hay indicios de la no correlación serial de los residuos medido por el Darwin Watson de 1,4 y además resultó significativo al $5 \%$ lo cual permite entender que reúne las características de homocedasticidad, autocorrelación y por ende la consistencia del modelo.

Seguidamente se pasa a presentar los resultados de las pruebas de cointegración lo cual se hace en la tabla $\mathrm{N}^{\circ} 4$.

Tabla $N^{\circ} 4$ Salidas de las pruebas de Cointegración Engle y Granger en las series de Inflación y Tasa de Interés Nominal 2005 2019

\begin{tabular}{ccccc}
\hline Dependent & tau-statistic & Prob. $^{\text { }}$ & z-statistic & Prob. $^{\text {* }}$ \\
\hline DTF & -3.292 .379 & 0.0695 & -2.187 .632 & 0.0219 \\
IPC & -3.697 .916 & 0.0273 & -2.840 .296 & 0.0035 \\
\hline
\end{tabular}

Fuente: Elaboración propia con datos de Banco de la Republica y Dane 2020.

La tabla $\mathrm{N}^{\circ} 4$ permite apreciar la integración de las series mediante la prueba de cointegración de Engle y Grange, demuestra que las dos variables en sus series están porque los componentes del vector $X$ están integrados de orden I(d). Lo cual se evidencia que el residual de la regresión es integrado de orden cero, además y de la significancia al $5 \%$ y algunos datos adicionales como lo más importante es la significancia a un nivel de confianza $5 \%$. Se puede afirmar según estos resultados que tanto la tasa de interés como la inflación pueden crecer en el tiempo y en especial en el largo plazo en forma uniforme de tal forma que no hay posibilidad de que el error de las dos series no crece. Podría entenderse como que la única fuente que haría cambiar los valores de la variable sería el tiempo sin que intervengan otras variables que puedan cambiar su comportamiento (Johansen 1988). 
La evidencia anterior del vector de cointegración entre la tasa de interés nominal y la inflación medida a través de DTF por haber dado un vector de cointegración I( 1) da la posibilidad de afirmar que el comportamiento de una variable es proporcional a la de la otra variable, es decir que si el IPC cambia en $1 \%$ la tasa de interés nominal DTF cambia en 1\% también. La inflación cambia y en esa misma proporción se transmite el cambio a la tasa de interés en el caso colombiano. Lo anterior puede considerarse una evidencia del efecto Fisher para Colombia. Es de anotar que solo son 61 observaciones y no hay seguridad si con más observaciones las cosas puedan cambiar.

El paso siguiente fue realizar la prueba de corrección de error cuyos resultados se presentan en la tabla 5.

\section{Tabla $N^{\circ} 5$ Prueba de corrección de error de la inflación y la tasa de interés nominal en Colombia 2005 - 2020.}

\begin{tabular}{ccccc}
\hline Variable & Coefficient & Std. Error & t-Statistic & Prob. \\
\hline RESID02(-1) & -0.429224 & 0.101373 & -4.234 .100 & 0.0001 \\
R-squared & 0.231699 & Mean dependent var & -0.051667 \\
$\quad$ Durbin-Watson & 2.040 .403 & & & \\
\hline
\end{tabular}

La prueba de corrección de error (Engle y Granger 1987) dio significativa a un nivel de confianza del $95 \%$ (Pvalue 0,0001), lo cual se interpreta que los cambios en la inflación se transmiten de manera conjunta en la tasa de interés de largo plazo, lo que corrobora lo dicho anteriormente en el sentido que los movimientos de los cambios del nivel general de precios se transmiten en cambios en la tasa de interés en el largo plazo lo que puede aceptarse con estos cálculos y los datos oficiales de domino publico que para Colombia aplica el efeto Fisher.

Comparando los resultados de este ejercicio con los de Ávila 2013, quien usó las mismas variables a excepción de un proxi con la metodología de series de tiempo y análisis de cointegración. Los resultados de la estimación del modelo son muy similares un coeficiente de determinación de 0,45, en el presente trabajo fue $\quad 0,46$ igual que en este trabajo las series fueron no estacionarias en niveles, pero si en primeras diferencias como también ocurrió en el trabajo de Cárdenas et al 2007 cuando pretendía demostrar la hipótesis de la neutralidad del dinero y el efecto Fisher. En los tres casos hubo presencia de raíces unitarias lo cual llevó a hacer las pruebas en primeras diferencias donde demostraron que eran estacionarias.

Los tres trabajos que se comparan con las mismas técnicas, pero en diferentes periodos de tiempo y en algunos casos además de la tasa de interés nominal y la inflación con otras variables como el Producto Interno Bruto PIB, el cual no se consideró en el presente trabajo llevan a resultados similares. La determinación de un comportamiento futuro por un movimiento presente en la inflación, en el trabajo de Ávila, por ejemplo, pudo probar la influencia que ejerce el ingreso expresado en el PIB y la inflación o comportamiento del nivel general de precios para explicar la demanda de dinero. Igualmente, Cárdenas eta al 2007 encontró que los cambios en la inflación en el largo plazo determinan la tasa de interés nominal. Lo que tienen en común los tres trabajos respecto a los resultados es que los aumentos de una variable en el largo plazo se transmiten a las demás variables siempre y cuando se trate de series estacionarias y pueden determinan su comportamiento en el tiempo como ocurrió en la relación de la tasa de interés nominal con la inflación en Colombia durante 14 años. Independiente de lo larga que sea la serie en los tres casos se llega a la misma conclusión.

\section{CONCLUSIONES:}

El comportamiento de una variable puede ser explicada a futuro por los aumentos de largo plazo de otra variable, lo cual permite evidenciar y validar hipótesis teóricas como la que se pretendió demostrar en la relación inflación, tasa de interés nominal donde quedó demostrado que los aumentos en la inflación influyen en el comportamiento futuro de la tasa de interés nominal.

Los herramientas y técnicas de análisis son un instrumento válido en la corroboración empírica de la 
teoría como quedó demostrado en el presente trabajo y en los estudios que sirvieron de referencia, para el caso colombiano. Por otra parte, la medición de la integración permite entender como existe transmisión de valores entre las variables macroeconómicas en este caso la evidencia del efecto Fisher la transmisión del efecto de la inflación en la tasa de interés.

Es muy conveniente hacer la advertencia en la prudencia y cuidado al tomar estos resultados por cuanto a pesar de que las técnicas fueron aplicadas con mucha rigurosidad y no hay duda de la construcción de las series por parte de instituciones oficiales que las ponen a disposición del público, aunque se pudo probar con estos métodos la transmisión de la inflación sobre la tasa de interés nominal es bueno advertir que de acuerdo con Friedman, los análisis deben ser de muy largo plazo y en nuestro caso pese a la consistencia de los resultados el periodo fue de 14 años que a mi juicio podría no ser suficiente al momento presentar los resultados como concluyentes. Agradecimientos. A la Corporación Colombiana de Investigación Agropecuaria AGROSAVIA por contarme dentro de su nómina de colaboradores como investigador PhD. A mis profesores de econometría en la Universidad Autónoma de Baja California UABC por sus enseñanzas.

\section{REFERENCIAS:}

Banco de la Republica (2020) Estadisticas monetarias, Tasas de captación de los CDT. Disponible en:https://www.banrep.gov.co/es/glosario/tas as-captacion

Dickey,D.A. y Fuller,W.A. (1981):" LikelihoodRatio statistics for Aytorregressive Time Series with a unit Root". Econometrica V 4
Dornbusch, R., Fischer, S.\&Startz, R. (2009). Macroeconomía. (10 ed.). México: McGraw-Hill.

Engle, R.F. y Granger, C.W.J.(1987) : "CoIntegration and Error Correction: Representation, Estimation and Testing". Econometrica 55.

Fisher, 1. [1930]. Tbe throry 01 interest, Macmillan, Nueva York.

Friedman, M. ; Schwartz, A. (1963). A Monetary History of the United States, 1867- 1960. New York:Edition Penguin Group.

Giorgetti, M., S. Calvo and L. Salvador, 2007. An analysis of the spatial integration of the soya and the corn markets. Agriscientia XXIV (2): 7985

Granger CWJ, Weiss AA. 1983. Time series analysis of error-correcting models. En: Karlin S, Amemuya T, Goodman LA, editores. Studies in econometrics, time series, and multivariate analysis. Nueva.

Hayek 1933 'El dinero neutral' On", en la FA Hayek. Dinero, el capital, y las fluctuaciones: Ensayos tempranas, editado por Roy McCloughry, Chicago

Johansen, S. 1988. Statistical analysis of cointegration vectors. Journal of Economic Dynamics and Control, 12:231254

Kates, H., Soltis, P. \& Soltis, D. (2017). Evolutionary and domestication history of Cucurbita (pumpkin and squash) species inferred from 44 nuclear loci. Molecular Phylogenetics and Evolution, 111, 98-109. 
Keynes, J. M. (1984). Teoría general de la ocupación, el interés y el dinero. México: Editado por el Fondo de Cultura Económica.

Superfinanciera 2020 Superintendencia financiera de Colombia. Estadísticas. Disponible en: (http://www.superfinanciera.gov.co/).

Uribe, J (1994). Inflación y Crecimiento Económico en Colombia: 1951-1992. En: Borradores de Economía 1\10 1 (Junio). Banco de la República, 18 p. 